\title{
Um modelo matemático para a interação de um vírus com a membrana plasmática da célula
}

\section{A mathematical model for the interaction of a virus with the plasma membrane of cell}

\author{
Diogo Martins Gonçalves de Morais \\ Faculdade de Tecnologia Termomecanica (FTT), São Bernardo do Campo, SP, Brasil \\ (D) https://orcid.org/0000-0001-5681-4044, pro7113@cefsa.edu.br
}

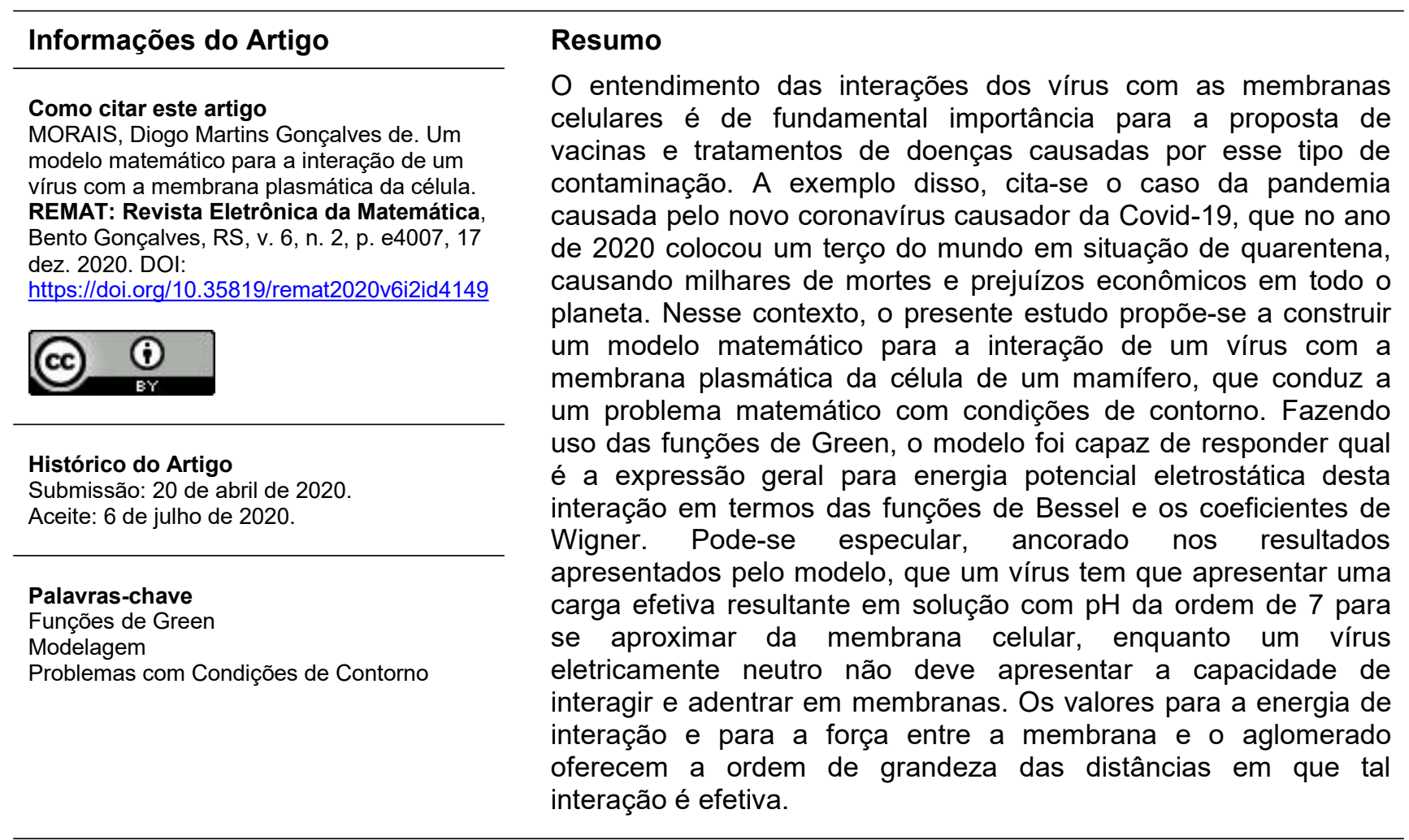

Keywords

Green's Functions

Modeling

Problems with Boundary Conditions

\begin{abstract}
Understanding the interactions of viruses with cell membranes is important for the proposal of vaccines and treatments for diseases caused by this type of contamination. An example of this is the case of the pandemic caused by the new Covid-19, which in 2020 placed was responsible for a third of the world under virus quarantine, causing huge economic losses and thousand of deaths across the planet. In this context, this research proposes to build a mathematical model for the interaction of a virus with the plasma membrane in a mammalian cell, which leads to a mathematical problem with boundary conditions. Using Green's functions, the model was able to answer what is the general expression for potential electrostatic energy of this interaction in terms of Bessel functions and Wigner coefficients. It is reasonable to speculate, based on the results presented by the model, that a virus has to present an effective charge resulting in a solution with a $\mathrm{pH}$ value of about 7 to approach the cell membrane, while an electrically neutral virus should not have the ability to interact and enter membranes. The values for the interaction energy and the force between the membrane and the cluster offer the order of magnitude of the distances over which such interaction is effective.
\end{abstract}




\section{Introdução}

No ano de 2020 o mundo foi surpreendido com uma das maiores epidemias causadas pelo novo coronavírus, chamado SARSCoV-2, que imobilizou em regime de quarentena aproximadamente um terço de todas as pessoas do mundo, impactando no sistema de saúde dos países e em suas economias. Nesse contexto, esforços de todas as áreas da ciência estão se juntando para atacar esse, que ainda hoje insiste em fragilizar a humanidade, que é a ação parasita dos vírus sobre as células humanas.

Para se fazer uma análise quantitativa de interações biomoleculares, deve-se sempre recorrer aos modelos simplificados, uma vez que a heterogeneidade estrutural das partículas envolvidas e a não homogeneidade do meio em que se encontram não permitem tratamento matemático simples. Ao considerar as propriedades de membranas celulares e suas moléculas constituintes, assim como as propriedades do meio eletrolítico em que se encontram, há a possibilidade de propor modelos que envolvem tratamento matemático para descrição quantitativa e análise de sistemas de interesse biológico.

Dessa maneira, o presente estudo tem o objetivo de apresentar um modelo matemático para descrever a interação de um vírus com a membrana plasmática da célula de um mamífero, a partir da modelo proposto por Morais (2005), para a interação de aglomerados moleculares imersos em soluções eletrolíticas, que conduz a um problema matemático com condições de contorno.

\section{Referencial teórico}

\subsection{Membrana plasmática}

De acordo com os estudos da biologia celular, todas as células possuem uma membrana (também chamada membrana plasmática), que separa o meio intracelular do extracelular e desempenha um papel importante no transporte de substâncias (ALBERTS et al., 2015). É sabido, também, que as células eucariotas possuem, além da membrana plasmática, um sistema de organelas constituintes de subsistemas celulares, tais como: núcleo, retículo endoplasmático, mitocôndrias, cloroplastos e complexo de Golgi (ALBERTS et al., 2015).

O mesmo autor assevera que as bases estruturais da membrana são constituídas por componentes lipídicos e protéicos. Os lipídios das membranas são moléculas anfipáticas, isto é, possuem uma extremidade hidrofílica (solúvel em meio aquoso) e uma cadeia hidrofóbica (insolúvel em meio aquoso). Entre os lipídios frequentes nas membranas celulares encontram-se fosfoglicerídios (fosfatidilcolina, fosfatidiletanolamina, fosfatidilserina e fosfatidiltreonina), esfingolipídios, glicolipídios e colesterol. Os fosfoglicerídios e os esfingolipídios contêm o radical fosfato e são chamados fosfolipídios. A Tabela 1 apresenta a composição lipídica de algumas membranas (ALBERTS et al., 2015). 
Tabela 1 - Composição lipídica (\%).

\begin{tabular}{c|c|c|c|c}
\hline Lipídio & $\begin{array}{c}\text { Membrana } \\
\text { plasmática } \\
\text { de célula de }\end{array}$ & $\begin{array}{c}\text { Membrana } \\
\text { plasmática de } \\
\text { glóbulo vermelho }\end{array}$ & Mitocôndria & $\begin{array}{c}\text { Retículo } \\
\text { endoplasmático }\end{array}$ \\
\hline Colesterol & 17 & 23 & 03 & 06 \\
\hline Fosfatidiletanolamina & 07 & 18 & 35 & 17 \\
\hline Fosfatidilserina & 04 & 07 & 02 & 05 \\
\hline Fosfatidilcolina & 24 & 17 & 39 & 40 \\
\hline Esfingomielina & 19 & 18 & 00 & 05 \\
\hline Glicolipídio & 07 & 03 & - & - \\
\hline Outros & 22 & 14 & 21 & 27 \\
\hline
\end{tabular}

Fonte: Adaptado de Alberts et al. (2015, p. 163).

Devido à natureza anfipática e à sua forma, os lipídios, quando em solução aquosa, e sob algumas condições, formam bicamadas. A Figura 1 ilustra a sua estrutura.

Figura 1 - llustração de uma bicamada lipídica.

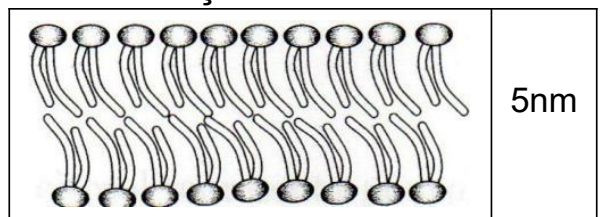

Fonte: Adaptado de Alberts et al. (2015, p. 79).

A parte hidrofóbica constitui o interior da bicamada e a extremidade hidrofílica mantém-se em contato com o meio aquoso.

\subsection{Meio celular}

Nas células eucariotas, segundo Alberts et al. (2015), o citosol representa pouco mais da metade de seu volume total e é o sítio de síntese e degradação de proteínas. É constituído de uma solução aquosa de pH 7,2, com elevada concentração de moléculas orgânicas, além de íons, como potássio, cloreto, magnésio, cálcio, sódio e fosfato. O autor assevera que a célula, como um todo, é eletricamente neutra, isto é, contém em seu interior mesmo número de cargas positivas e negativas. A Tabela 2 mostra a composição iônica de uma célula típica de mamífero, embora a célula contenha muito outros ânions e cátions não listados, tais como $\mathrm{HCO}_{3}^{-}$(bicarbonato), $\mathrm{PO}_{4}^{-3}$ (fosfato), ácidos nucleicos, proteínas, etc. 
Tabela 2 - Concentração iônica de uma célula típica de mamífero.

\begin{tabular}{c|c|c}
\hline Componente & Concentração intracelular $(\mathbf{m M})$ & Concentração extracelular (mM) \\
\hline Cátions & & 145 \\
\hline $\mathrm{N \alpha}^{+}$ & $5-15$ & 5 \\
\hline $\mathrm{K}^{+}$ & 140 & $1-2$ \\
\hline $\mathrm{Mg}^{2+}$ & 0,5 & $1-2$ \\
\hline $\mathrm{Ca}^{2+}$ & $10^{-4}$ & $4.10^{-5}$ \\
\hline $\mathrm{H}^{+}$ & $7.10^{-5}$ & \\
\hline$\hat{A}^{n}$ ions & & 110 \\
\hline $\mathrm{ICl}$ & $5-15$ & \\
\hline
\end{tabular}

Fonte: Adaptado de Alberts et al. (2015, p. 98).

Devido à equivalência de força iónica, eletrólitos de aproximadamente $150 \mathrm{mM}$ de íons monovalentes são usados frequentemente para simular o meio celular.

\subsection{O meio celular e o equilíbrio eletrostático}

No meio celular, é possível verificar em que condições o sistema estaria em equilíbrio eletrostático, lembrando, é claro, que o equilíbrio do sistema como um todo é devido a outros fatores além da interação eletromagnética. Segundo Jackson (1998), o tempo de relaxação de um sistema sob perturbação de um campo elétrico externo ou de uma variação nos valores da densidade de cargas pode ser avaliado, a partir da equação de continuidade:

$$
\nabla \cdot \vec{J}+\frac{\partial \rho}{\partial t}=0
$$

$\mathrm{Na}$ equação acima, $\vec{J}$ representa o vetor densidade de corrente. O princípio em que repousa a equação é o princípio da conservação da carga elétrica.

Se o meio que constitui o sistema apresenta características ôhmicas e isotrópicas, o vetor densidade de corrente é escrito, de acordo com Jackson (1998), como:

$$
\vec{J}=\sigma \vec{E}
$$

As grandezas $\rho, \vec{J}$ e $\vec{E}$ descrevem perturbações ao sistema e $\sigma$ representa a condutividade característica do meio.

Usando a lei de Gauss $\nabla \vec{E}=4 \pi \frac{\rho}{\epsilon}$, temos que a equação (1) se apresenta como: 


$$
\frac{\partial \rho}{\partial t}=-4 \pi \frac{\sigma}{\epsilon} \rho
$$

A solução da equação acima é dada por $\rho(t)=\rho(0) e^{\left(-4 \pi \frac{\sigma}{\epsilon} t\right)}$ (JACKSON, 1998). Podemos, então, observar que a perturbação $\rho(t)$ decai com um tempo de relaxação $\tau=\frac{\epsilon}{4 \pi \sigma}$, sendo $\epsilon$ o valor da constante dielétrica do meio (JACKSON, 1998).

Se considerarmos um eletrólito, composto por $100 \mathrm{mM}$ de $\mathrm{NaCl}$, temos que

$$
\sigma=\Lambda_{m} c=\left(v_{+} \lambda_{+}+v_{-} \lambda_{-}\right) v=1,26 S / m=1,13.10^{10} s^{-1}
$$

onde $\Lambda_{m}$ é a condutividade molar e $c$ a concentração do sal. Os valores da condutividade dos cátions $\left(\lambda_{+}\right)$e dos ânions $\left(\lambda_{-}\right)$, assim como seus respectivos coeficientes estequiométricos, são dados na Tabela 3.

Tabela 3 - Condutividade iônica na água a $25^{\circ} \mathrm{C}$.

\begin{tabular}{c|c|c}
\hline $\mathbf{N a C l}$ & $v$ & $\boldsymbol{\lambda}\left(\mathbf{m S m}^{2} \mathbf{m o l}^{-1}\right)$ \\
\hline $\mathrm{N \alpha}^{+}$ & 1 & 5,01 \\
\hline $\mathrm{Cl}^{-}$ & 1 & 7,63 \\
\hline
\end{tabular}

Fonte: Adaptado de Atkins, Paula e Keeler (2018, p. 112).

Uma estimativa do tempo de relaxação é obtida atribuindo a $\epsilon$ o valor da constante dielétrica da água a $25^{\circ} \mathrm{C}$, ou seja, $\epsilon=78,5$, e considerando o valor da condutividade característica $\left(1,13 \times 10^{10} \mathrm{~s}^{-1}\right)$ do eletrólito, o que nos permite concluir que o tempo é extremamente pequeno, dado por $\tau=5,5 \times 10^{-10} \mathrm{~s}$ (ATKINS; PAULA; KEELER, 2018).

Desta maneira, esse estudo sugere que o tempo de relaxação do sistema após uma perturbação seja ainda menor, quando forem consideradas soluções que simulam meios fisiológicos, onde a concentração de sais é maior ou igual a 100 mM.

As informações e números apresentados permitem tratar eventos como o deslocamento de aglomerados moleculares imersos em soluções de eletrólitos, como é o caso de vírus e membranas celulares, com o campo e as cargas em equilíbrio eletrostático.

\subsection{Equação de Poisson-Boltzmann}

Considerando um meio linear e isotrópico, com constante dielétrica $\epsilon$ e densidade de cargas $\rho$, temos que a equação que relaciona potencial eletrostático e densidade de cargas é a equação de Poisson (HILL, 1987):

$$
\nabla^{2} \Psi(\vec{r})=-4 \pi \frac{\rho(\vec{r})}{\epsilon}
$$


Os símbolos têm significado usual, sendo $\nabla^{2}$ o operador laplaciano.

Ao tratarmos um sistema composto por uma solução iônica, temos que a densidade de cargas $\rho$ será dada pela soma das densidades das espécies iônicas presentes no sistema (HILL, 1987):

$$
\rho(\vec{r})=\sum_{i} z_{i} e n_{i}(\vec{r})
$$

O parâmetro $z_{i}$ representa a valência do íon, e representa o valor da carga do elétron e $n_{i}$ o número de íons da espécie $i$ por unidade de volume.

Admitindo que toda interação possa ser representada pelo potencial eletrostático médio, temos para o potencial químico da espécie iônica $i$, em qualquer parte do sistema (HILL, 1987):

$$
\mu_{i}(\vec{r})=\mu_{0 i}+k T \ln \left(n_{i}(\vec{r})\right)+z_{i} e \Psi(\vec{r})
$$

O termo $\mu_{0 i}$ compreende a contribuição de uma molécula ou íon atômico no que se refere às suas propriedades intrínsecas, em termos de valores médios (graus de liberdade vibracional, rotacional e translacional, estados nucleares e eletrônicos, sendo que os dois últimos, via de regra, são tomados no estado fundamental). O termo $k \operatorname{Tn}\left(n_{i}\right)$ compreende a contribuição entrópica coletiva, sendo $k$ a constante de Boltzmann, dada por $k=1,38 \times 10^{-16}$ ergs $\times \mathrm{K}^{-1}$ e $z_{i} e \Psi(\vec{r})$ a contribuição dada pela interação eletrostática.

Considerando duas regiões distintas do sistema designadas por $\overrightarrow{r_{0}}$ e $\vec{r}$, e atribuindo o valor $\Psi\left(\overrightarrow{r_{0}}\right)=0$, podemos escrever:

$$
\begin{gathered}
\mu_{i}\left(\overrightarrow{r_{0}}\right)=\mu_{0 i}+k T \ln \left(n_{i 0}\left(\overrightarrow{r_{0}}\right)\right) \\
\mu_{i}(\vec{r})=\mu_{0 i}+k T \ln \left(n_{i}(\vec{r})\right)+z_{i} e \Psi(\vec{r})
\end{gathered}
$$

Admitindo o equilíbrio termodinâmico, temos que o potencial químico é o mesmo para regiões quaisquer do sistema, isto é, $\mu_{i}\left(\overrightarrow{r_{0}}\right)=\mu_{i}(\vec{r})$. Logo, obtemos:

$$
n_{i}(\vec{r})=n_{i 0} e^{\left(\frac{-z_{i} e \Psi(\vec{r})}{k T}\right)}
$$

conhecida como distribuição de Boltzmann para espécies iônicas, sendo $n_{i 0}$ a concentração de íons da espécie $i$ onde o potencial eletrostático médio é nulo. Substituindo (10) em (6), segue que:

$$
\rho(\vec{r})=\sum_{i} z_{i} e n_{i 0} e^{\left(\frac{-z_{i} e \Psi(\vec{r})}{k T}\right)}
$$


que é a densidade de cargas em termos das cargas dos íons, do potencial eletrostático médio, da temperatura e da concentração de íons por unidade de volume onde o potencial eletrostático médio é nulo.

Substituindo (11) em (5), obtemos (HILL, 1987):

$$
\nabla^{2} \Psi(\vec{r})=-\sum_{i} \frac{4 \pi z_{i} e n_{i 0}}{\epsilon} e^{\left(\frac{-z_{i} e \Psi(\vec{r})}{k T}\right)}
$$

Essa é uma equação diferencial parcial não linear conhecida como equação de PoissonBoltzmann. Sua solução analítica existe para casos muito específicos, como sistemas dotados de simetria cilíndrica com uma espécie iônica na solução e sistemas com simetria plana contendo misturas de íons mono e bivalentes (TESO; DRIGO FILHO; AGOSTINHO NETO, 1997).

\subsection{Aproximação de Debye-Hückel}

O efeito da força iônica do meio pode ser tratado por meio da teoria de Debye Hückel, a qual permite a aplicação da equação de Poisson-Boltzmann a sistemas que podem ser adequadamente representados como um conjunto de cargas em um meio de constante dielétrica uniforme e de baixa força iônica, de forma que a energia potencial eletrostática $z_{i} e \Psi(\vec{r})$ em qualquer ponto da solução seja desprezível em relação a energia térmica $k T$ (HILL, 1987).

Em tais condições é possível tratar linearmente a distribuição iônica representada pelo fator exponencial da equação (12). A linearização da equação é feita por meio da expansão em série de Taylor do termo exponencial, e da utilização apenas do termo de primeira ordem (HILL, 1987):

$$
\mathrm{e}^{\left(\frac{-\mathrm{z}_{\mathrm{i}} \mathrm{e} \Psi(\overrightarrow{\mathrm{r}})}{\mathrm{kT}}\right)} \approx\left(1-\frac{\mathrm{z}_{\mathrm{i}} \mathrm{e} \Psi(\overrightarrow{\mathrm{r}})}{\mathrm{kT}}\right)
$$

Substituindo então a relação (13) na equação (12), obtemos (HILL, 1987):

$$
\nabla^{2} \Psi(\vec{r})=-\sum_{i} \frac{4 \pi z_{i} e n_{i 0}}{\epsilon}+\sum_{1} \frac{4 \pi\left(z_{i} e\right) n_{i 0}}{\epsilon k T} \Psi(\vec{r})
$$

Devido à condição de eletroneutralidade da solução eletrolítica, o somatório do primeiro termo se anula $\left(\sum_{i} \frac{4 \pi z_{i} e n_{i 0}}{\epsilon}=0\right)$. Nestas condições, a equação (14) reduz-se à equação de Helmholtz modificada (HILL, 1987),

$$
\nabla^{2} \Psi(\vec{r})=\kappa^{2} \Psi(\vec{r})
$$

cuja solução geral pode ser escrita em termos de funções conhecidas. 
O fator $\kappa^{-1}=\left(\frac{\epsilon k T}{\Sigma_{1} 4 \pi\left(z_{1} e\right)^{2} n_{i 0}}\right)$ é denominado comprimento de Debye, e está relacionado com a efetividade da blindagem produzida pela solução eletrolítica Debye (HILL, 1987). A Tabela 4 apresenta a espessura da atmosfera iônica para soluções com diferentes concentrações de eletrólito.

Tabela 4 - Comprimento de Debye para algumas concentrações.

\begin{tabular}{c|c}
\hline Concentração de eletrólito (1:1) & Espessura da atmosfera iônica \\
\hline $1 \mathrm{mM}$ & $96,4 \AA$ \\
\hline $10 \mathrm{mM}$ & $30,5 \AA$ \\
\hline $100 \mathrm{mM}$ & $9,64 \AA$ \\
\hline $150 \mathrm{mM}$ & $7,94 \AA$ \\
\hline
\end{tabular}

Fonte: Adaptado de Hill (1987, p. 129).

Registra-se que o aumento da concentração salina causa um decréscimo nessa grandeza, sugerindo que podemos interpretá-la como um parâmetro de escala que indica a distância em que o potencial produzido por um íon é efetivamente blindado.

\section{Metodologia}

A seguir, será apresentado o percurso metodológico para a obtenção do modelo que se propõe construir, por meio das aproximações e simplificações usadas, obtendo assim um modelo para a interação de uma membrana celular e um vírus. A Figura 2 ilustra o percurso metodológico do estudo.

Figura 2 - Percurso metodológico do estudo.

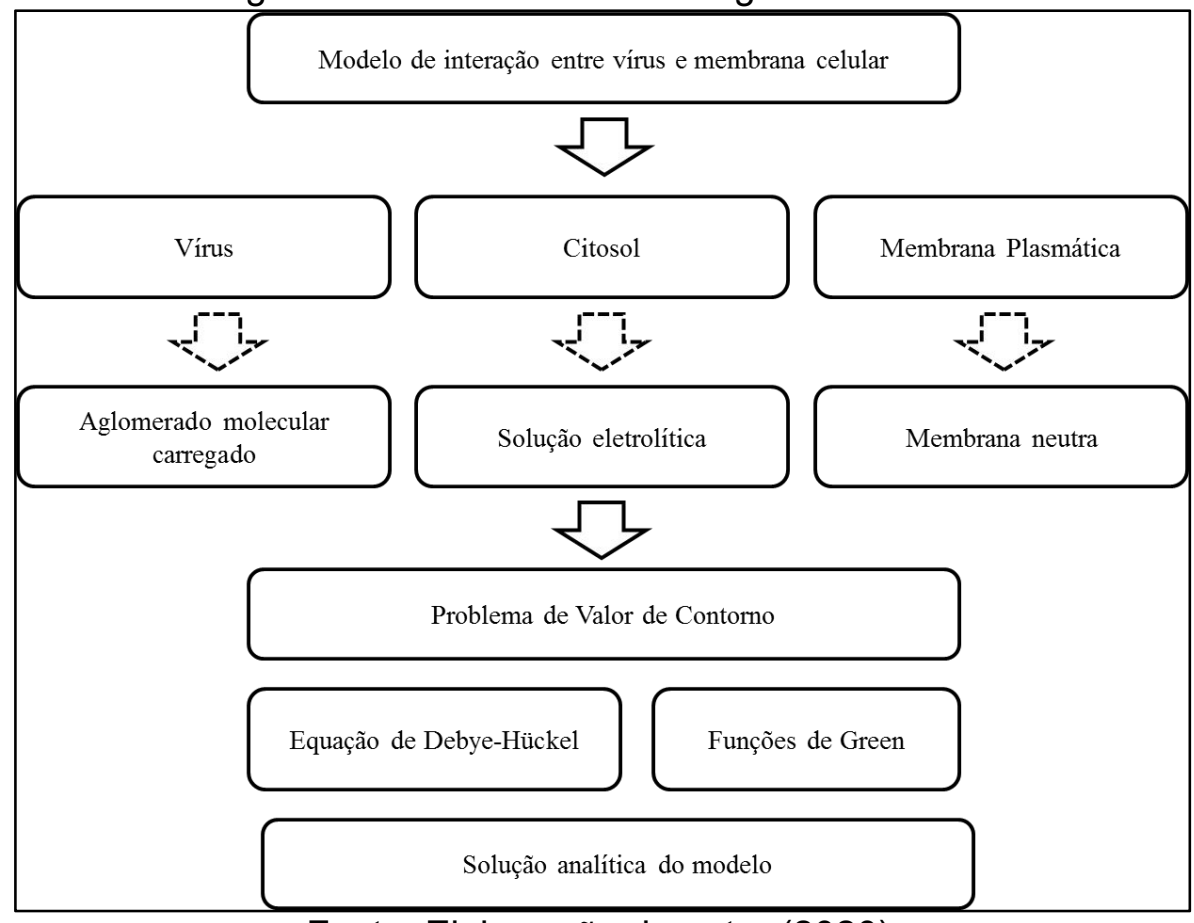

Fonte: Elaboração do autor (2020). 
O meio celular, ou seja, o citosol, será tratado como uma solução eletrolítica, isto é, como um dielétrico contínuo e os íons da solução pelo seu comportamento médio, além de considerar que sua distribuição é descrita pela estatística de Boltzmann ao redor de um aglomerado molecular carregado imerso na solução.

Feitas essas primeiras considerações, temos que o potencial eletrostático médio em todo o sistema, admitindo o equilíbrio termodinâmico, satisfaz a equação de Poisson-Boltzmann (12).

A fim de obter uma solução matemática analítica para o problema de interesse, será considerada a aproximação de Debye-Hückel para o sistema. Essa consideração faz com que o potencial eletrostático médio seja solução de uma equação diferencial parcial linear de Helmholtz modificada (15), sendo escrita em termos de funções conhecidas.

Registra-se que, em determinadas concentrações e em certas distâncias do aglomerado carregado, o potencial eletrostático médio obtido da equação de Poisson-Boltzmann e DebyeHückel são equivalentes, como demonstrado em outros estudos, como por Morais (2005), e por Teso, Drigo Filho e Agostinho Neto (1997).

Uma simplificação feita para a análise do comportamento das membranas biológicas é considerar membranas compostas apenas por fosfolipídios. Os números apresentados no referencial teórico a respeito da composição fosfolipídica de algumas células de mamíferos (Tabela 1) e sua estrutura química (Figura 1) nos sugere tratar membranas fosfolipídicas imersas em solução eletrolítica como sendo superfícies eletricamente neutras e condutoras. Isso se justifica pelo fato de ter íons dissociados na estrutura dos principais fosfolipídios, fazendo com que contraíons constituintes da solução eletrolítica sejam adsorvidos na membrana.

Fazendo tais considerações a respeito da membrana e da solução eletrolítica, tem-se que a interação entre um vírus, considerado um aglomerado molecular carregado, e a membrana, imersos em solução, será sempre atrativa.

Quando o vírus, ou seja, o aglomerado for carregado positivamente, tem-se uma densidade de cargas induzida negativa e vice-versa. A formação da densidade de cargas induzida na membrana será sempre feita pela atração ou repulsão dos contraíons adsorvidos na membrana, dependendo da carga do aglomerado molecular com o qual está interagindo.

\section{Resultados e discussão}

Considerando a interação eletrostática de um aglomerado molecular com distribuição de carga superficial dada por $\sigma\left(\widehat{r_{0}}\right)$ e uma superfície condutora neutra, plana e infinita, ambos imersos em solução eletrolítica (Figura 3), é possível obter o potencial eletrostático em uma posição $\vec{r}$ do meio, relacionado a $\sigma\left(\widehat{r_{0}}\right)$, juntamente com a densidade de cargas induzida na superfície condutora. 
Figura 3 - llustração do problema de valor de contorno.

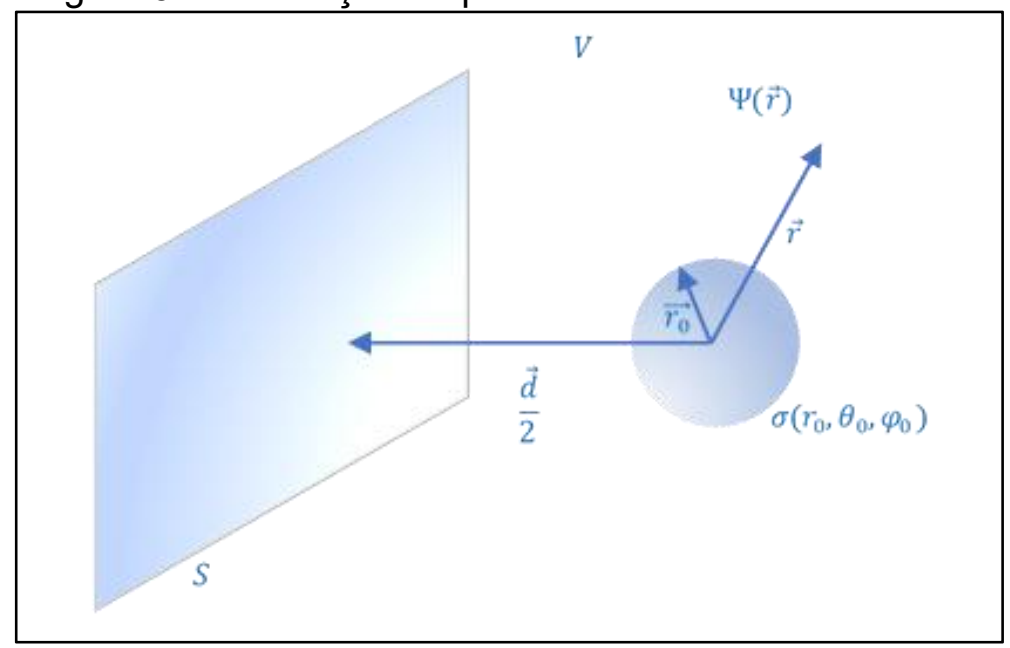

Fonte: Elaboração do autor (2020).

Como esse potencial satisfaz a equação de Helmholtz modificada (15), tem-se o seguinte problema de valor de contorno:

$$
\begin{gathered}
\nabla^{2} \psi(\vec{r})-\kappa^{2} \psi(\vec{r})=0, \forall \vec{r} \in V \\
\psi(\vec{r} \in S)=0
\end{gathered}
$$

A solução do problema de valor de contorno será feita por meio do uso da função de Green, que será encontrada com o uso do método das imagens.

\subsection{Funções de Green para o problema de valor de contorno}

Se $G\left(\vec{r} \backslash \overrightarrow{r_{0}}\right)$ for o campo gerado no ponto de observação $\vec{r}$ por uma fonte pontual unitária na posição $\overrightarrow{r_{0}}$, então o campo gerado em $\vec{r}$ por uma distribuição fonte $\sigma\left(\overrightarrow{r_{0}}\right)$ é a soma de $G\left(\vec{r} \backslash \overrightarrow{r_{0}}\right)$ sobre todo o espaço de $\overrightarrow{r_{0}}$ ocupado pela fonte. A função $G \sigma$ é chamada função influência ou função de Green (FESHBACH; MORSE; MICHIO, 2019).

A função de Green que descreve o potencial eletrostático devido a uma carga elétrica puntiforme imersa em uma solução eletrolítica, satisfaz a equação não homogênea (FESHBACH; MORSE; MICHIO, 2019, p. 68):

$$
\nabla_{\vec{r}}^{2} G_{\kappa}\left(\vec{r} \backslash \overrightarrow{r_{0}}\right)-\kappa^{2} G_{\kappa}\left(\vec{r} \backslash \overrightarrow{r_{0}}\right)=-\frac{4 \pi}{\epsilon} \delta\left(\vec{r}-\overrightarrow{r_{0}}\right)
$$

Os símbolos têm significado usual, ou seja, o operador laplaciano é representado por $\nabla^{2}$, $\kappa^{-1}$ é o comprimento de Debye, $\delta\left(\vec{r} \backslash \overrightarrow{r_{0}}\right)$ é a função delta de Dirac e $\epsilon$ a constante dielétrica do meio. 


\subsubsection{Funções de Green: contorno no infinito}

Para resolver problemas em que as superfícies de contorno estão no infinito, a função de Green só depende de $R=\left|\vec{r}-\overrightarrow{r_{0}}\right|$ e é esfericamente simétrica. Usando o Laplaciano em coordenadas esféricas, temos que $g_{k}(R)$ satisfaz:

$$
\frac{1}{R^{2}} \frac{d}{d R}\left(R^{2} \frac{d}{d R}\right) g_{k}(R)-k^{2} g_{k}(R)=-\frac{4 \pi}{\epsilon} \delta(\vec{R})
$$

Exceto para $\vec{r}=\overrightarrow{r_{0}}, R g_{\kappa}(R)$ satisfaz a equação homogênea

$$
\frac{d^{2}}{d R^{2}}\left(R g_{\kappa}(R)\right)-\kappa^{2}\left(R g_{\kappa}(R)\right)=0
$$

cuja solução é dada por $R g_{k}(R)=A e^{-k R}+B e^{k R}$.

Para $R \rightarrow \infty$, temos que $g_{\kappa}(R)$ se anula, e para $R \rightarrow 0$ a singularidade $\frac{1}{\epsilon R}$, uma vez que o potencial tem comportamento coulombiano. Portanto, tem-se que $A=1 / \epsilon$ e $B=0$ obtendo, então, a solução para a Equação (17), dada por $G_{\kappa}\left(\vec{r} \backslash \overrightarrow{r_{0}}\right)=g_{\kappa}\left(\vec{r} \backslash \overrightarrow{r_{0}}\right)=\frac{e^{-\kappa\left|\vec{r}-\overrightarrow{r_{0}}\right|}}{\epsilon\left|\vec{r}-\overrightarrow{r_{0}}\right|}$.

Nestas condições, o potencial eletrostático $\psi(\overrightarrow{\mathrm{r}})$ devido à distribuição de cargas superficial $\sigma\left(\widehat{r_{0}}\right)$ de um aglomerado molecular imerso em solução eletrolítica, tal que $\nabla^{2} \psi(\vec{r})-k^{2} \psi(\vec{r})=0$, é dado por:

$$
\psi(\vec{r})=\oint_{S_{0}} \frac{e^{-k\left|\vec{r}-\overrightarrow{r_{0}}\right|}}{\epsilon\left|\vec{r}-\overrightarrow{r_{0}}\right|} \sigma\left(\widehat{r_{0}}\right) d S_{0}
$$

\subsubsection{Funções de Green: contorno na superfície}

Com contorno no infinito, $\left.g_{k}(\vec{r}\rangle \overrightarrow{r_{0}}\right)$ é a função de Green apropriada. Em eletrostática, quando uma superfície de contorno é introduzida, uma contribuição ao potencial eletrostático passa a existir devido a carga induzida na superfície.

Assim, podemos esperar que $G_{k}\left(\vec{r} \backslash \overrightarrow{r_{0}}\right)=g_{k}\left(\vec{r} \backslash \overrightarrow{r_{0}}\right)+F_{k}\left(\vec{r} \backslash \overrightarrow{r_{0}}\right)$, onde $F_{k}\left(\vec{r} \backslash \overrightarrow{r_{0}}\right)$ representa os efeitos do contorno. $F_{k}\left(\vec{r} \backslash \overrightarrow{r_{0}}\right)$ não pode ter uma singularidade dentro da região considerada, de forma que, quando $\vec{r} \rightarrow \overrightarrow{r_{0}}, G_{k}\left(\vec{r} \backslash \overrightarrow{r_{0}}\right)=g_{k}\left(\vec{r} \backslash \overrightarrow{r_{0}}\right)$. Para obter $F_{k}\left(\vec{r} \backslash \overrightarrow{r_{0}}\right)$, usou-se o método das imagens, apresentado a seguir.

\subsubsection{O potencial eletrostático da interação}

Considerando o problema de valor de contorno ilustrado na Figura 3, dado por:

$$
\begin{gathered}
\nabla^{2} \psi(\vec{r})-\kappa^{2} \psi(\vec{r})=0, \forall \vec{r} \in V \\
\psi(\vec{r} \in S)=0
\end{gathered}
$$


Admitindo que exista uma distribuição de cargas, caracterizada pela densidade $\sigma\left(\widehat{r_{0}}\right)$, inteiramente situada na região externa a $V$, porém com posição simétrica à distribuição em $V$ e tal que, juntas, produzam nos pontos da fronteira feitos pela superfície condutora plana, o mesmo potencial nulo prescrito pela condição de contorno do problema considerado.

Dessa forma, ao nosso problema de valor de contorno (Figura 4 - Situação (a)), fazemos corresponder um outro, que denominaremos problema associado (Figura 4 - Situação (b)), sem fronteiras e com a seguinte distribuição de cargas: $\sigma\left(\widehat{r_{0}}\right)$ em $V$ e $\sigma\left(\widehat{r_{0}^{\prime}}\right)$ em $V^{\prime}$.

Figura 4 - Problema de valor de contorno: situação (a) e situação (b).

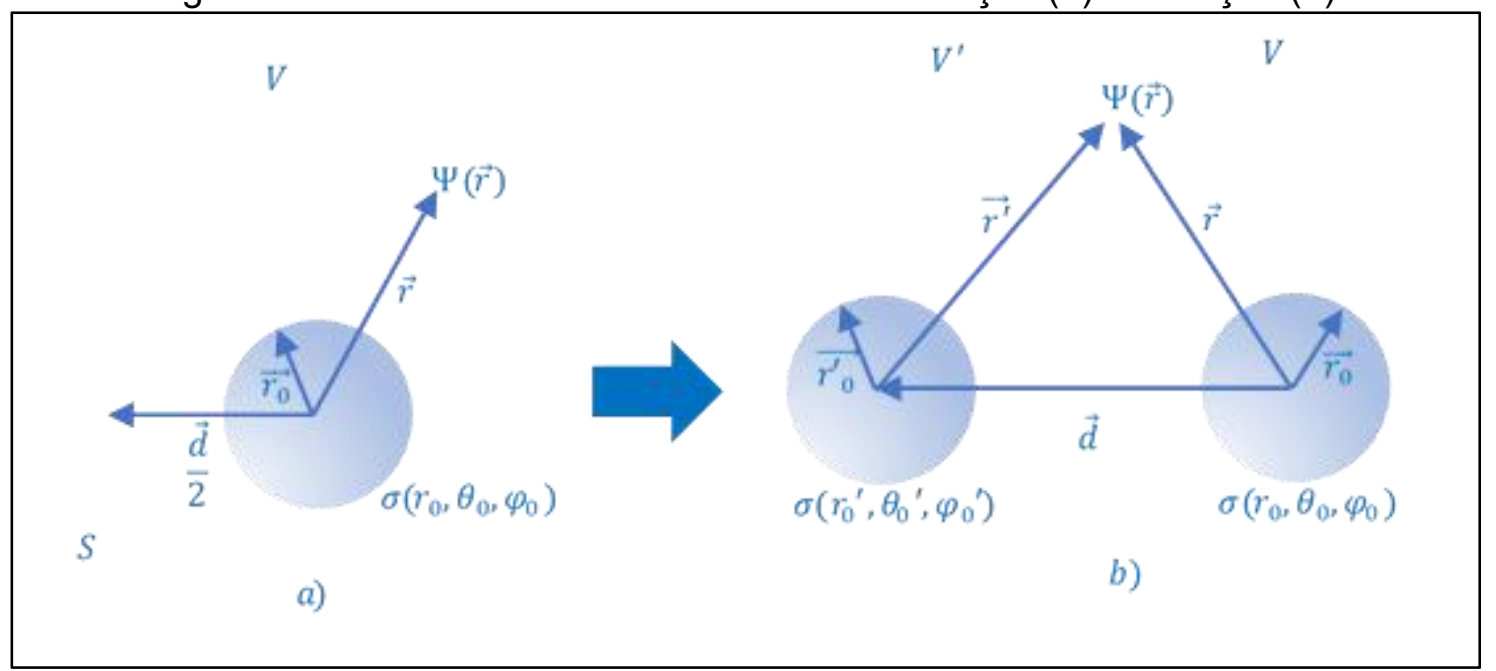

Fonte: Elaboração do autor (2020).

Visto que, para o problema associado é conhecida a distribuição de cargas em todo o espaço, temos a seguinte solução para o potencial do problema associado:

$$
\psi(\overrightarrow{\mathrm{r}})=\oint_{S_{0}} \frac{\mathrm{e}^{-\mathrm{k}\left|\overrightarrow{\mathrm{r}}-\overrightarrow{\mathrm{r}_{0}}\right|}}{\epsilon \mid \overrightarrow{\mathrm{r}}-\overrightarrow{\mathrm{r}_{0} \mid}} \sigma\left(\widehat{\mathrm{r}_{0}}\right) \mathrm{dS}_{0}+\oint_{\mathrm{S}_{0}^{\prime}} \frac{\mathrm{e}^{-\mathrm{k}\left|\overrightarrow{\mathrm{r}^{\prime}}-\overrightarrow{\mathrm{r}_{0}}\right|}}{\epsilon\left|\overrightarrow{\mathrm{r}}-\overrightarrow{\mathrm{r}_{0}}\right|} \sigma\left(\widehat{\mathrm{r}_{0}}\right) \mathrm{dS}_{0}^{\prime}, \forall \overrightarrow{\mathrm{r}} \in V U \mathrm{~V}^{\prime}
$$

onde $\overrightarrow{r^{\prime}}=\vec{r}-\vec{d}, \sigma\left(\widehat{r_{0}^{\prime}}\right)=-\sigma\left(\widehat{r_{0}}\right)$ e $V^{\prime}$ a região do espaço complementar a $V$.

É possível observar que $\psi(\vec{r})$ é tal que $\nabla^{2} \psi(\vec{r})-k^{2} \psi(\vec{r})=0, \forall \vec{r} \in V$, visto que $\sigma\left(\widehat{r_{0}}\right)=0$ para $\vec{r} \in V$ e ainda que $\psi(\vec{r})=0$, para $\vec{r}$ localizado na superfície condutora plana, por construção do problema associado. Portanto, para $\vec{r}$ restrito a $V$, temos:

$$
\psi(\vec{r})=\oint_{S_{0}} \frac{e^{-k\left|\vec{r}-\overrightarrow{r_{0}}\right|}}{\epsilon\left|\vec{r}-\overrightarrow{r_{0}}\right|} \sigma\left(\widehat{r_{0}}\right) d S_{0}+\oint_{S_{0}^{\prime}} \frac{e^{-k\left|\vec{r}-\overrightarrow{r_{0}}\right|}}{\epsilon\left|\vec{r}-\overrightarrow{r_{0}^{\prime}}\right|} \sigma\left(\widehat{r_{0}^{\prime}}\right) d S_{0}^{\prime}, \forall \vec{r} \in V
$$

O método acima exposto, que consiste em achar a solução de um problema de valor de contorno com condições de contorno de Dirichlet resolvendo um problema sem fronteiras com 
uma distribuição adicional $\sigma\left(\widehat{r_{0}^{\prime}}\right)$ na região $V^{\prime}$ externa a $V$ é chamado método das imagens, sendo que $F_{k}\left(\vec{r} \backslash \overrightarrow{r_{0}}\right)=\frac{e^{-k\left|\vec{r}-\overrightarrow{r_{0}}\right|}}{\epsilon\left|\vec{r}-\overrightarrow{r_{0}}\right|}$.

Registra-se que a função de Green $G_{k}\left(\vec{r} \backslash \overrightarrow{r_{0}}\right)=\frac{e^{-k\left|\vec{r}-\overrightarrow{r_{0}}\right|}}{\epsilon\left|\vec{r}-\overrightarrow{r_{0}}\right|}+\frac{e^{-k\left|\vec{r}-\overrightarrow{r_{0}}\right|}}{\epsilon\left|\vec{r}-\overrightarrow{r_{0}}\right|}$, encontrada para a interação do vírus com a membrana, é a mesma usada na teoria clássica de interações entre macroíons esféricos, apresentada por Verwey e Overbeek (1948).

\subsubsection{A energia potencial eletrostático da interação}

Sendo os efeitos da densidade de carga induzida na superfície condutora plana bem representados por $\sigma\left(\widehat{r_{0}^{\prime}}\right)$ do problema associado, pode-se partir para o cálculo da energia potencial eletrostática do problema de interesse.

Para isso, calcula-se o potencial eletrostático devido a soma das cargas em $V^{\prime}$ nas posições das cargas em $V$, ou seja, faz-se $\vec{r}=\overrightarrow{r_{0}}$. A Figura 5 ilustra esta situação.

Figura 5 - Obtenção da energia potencial eletrostática da interação.

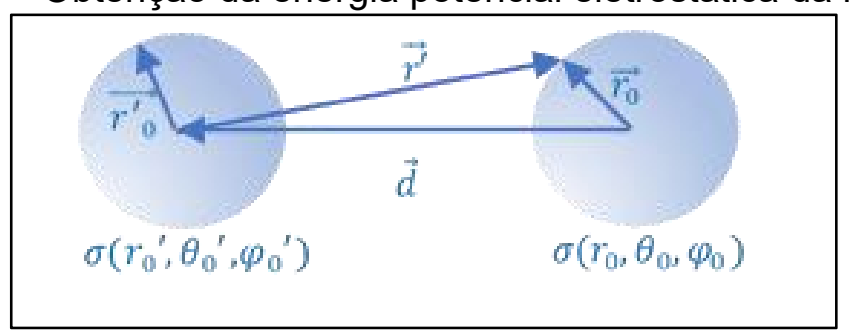

Fonte: Elaboração do autor (2020).

Dessa forma, obtém-se a energia potencial eletrostática da interação dos dois aglomerados carregados:

$$
U=\oint_{S_{0}} \oint_{S_{0}^{\prime}} \frac{e^{-k\left|\vec{r}-\overrightarrow{r_{0}}\right|}}{\epsilon\left|\overrightarrow{r^{\prime}}-\overrightarrow{r_{0}^{\prime}}\right|} \sigma\left(\widehat{r_{0}^{\prime}}\right) d S_{0}^{\prime} \sigma\left(\widehat{r_{0}}\right) d S_{0}
$$

\subsection{A solução do modelo em termos das funções de Bessel e coeficientes de Wigner}

Sendo $\frac{e^{-k\left|\vec{r}-\overrightarrow{r_{0}}\right|}}{\epsilon \vec{r}-\overrightarrow{r_{0}} \mid}$ a função de Green para o problema da interação de um vírus com a membrana celular, pode-se demonstrar que esta função pode ser expandida com o uso dos harmônicos esféricos, como demonstrado por Morais (2005), de modo que

$$
G_{\kappa}\left(\vec{r} \backslash \overrightarrow{r_{0}}\right)=\frac{e^{-k\left|\vec{r}-\overrightarrow{r_{0}}\right|}}{\epsilon\left|\overrightarrow{r^{\prime}}-\overrightarrow{r_{0}^{\prime}}\right|}=\sum_{n=0}^{\infty} \sum_{m=-n}^{n} \frac{8 \kappa}{\epsilon} i_{n}\left(\kappa\left|\overrightarrow{r_{0}}\right|\right) k_{n}(\kappa|\vec{r}|) Y_{n m}^{*}\left(\theta_{0}, \phi_{0}\right) Y_{n m}\left(\theta_{0}, \phi_{0}\right)
$$


Substituindo a Equação (25) na relação para a energia potencial eletrostática da interação entre vírus e proteína, dada pela Equação (24), tem-se que:

$$
U=\oint_{S_{0}} \sum_{n=0}^{\infty} \sum_{m=-n}^{n} \frac{8 k}{\epsilon} i_{n}\left(k\left|\overrightarrow{r_{0}^{\prime}}\right|\right) k_{n}\left(k\left|\overrightarrow{r^{\prime}}\right|\right) Y_{n m}\left(\widehat{r^{\prime}}\right) \sigma\left(\widehat{r_{0}}\right) d S_{0} \oint_{S_{0}} \sigma\left(\widehat{r_{0}^{\prime}}\right) Y_{n m}^{*}\left(\widehat{r_{0}^{\prime}}\right) d S_{0}^{\prime}
$$

Lembrando que no modelo de interação de um vírus com a membrana, $\overrightarrow{\mathrm{r}}=\overrightarrow{\mathrm{r}_{0}}-\overrightarrow{\mathrm{d}}$, é possível demonstrar que $k_{n}\left(k\left|\overrightarrow{r^{\prime}}\right|\right) Y_{n m}\left(\widehat{r^{\prime}}\right)=k_{n}\left(k\left|\overrightarrow{r_{0}}-\vec{d}\right|\right) Y_{n m}\left(\overrightarrow{r_{0}}-\vec{d}\right)$ pode ser escrito em termos dos coeficientes de Wigner, utilizados em alguns estudos da Mecânica Quântica, como apresentado por Messiah (1995), o que possibilita obter no presente estudo, em particular, a seguinte relação:

$$
\begin{aligned}
& Y_{n m}\left(\overrightarrow{r_{0}}-\vec{d}\right) k_{n}\left(\kappa\left|\overrightarrow{r_{0}}-\vec{d}\right|\right)= \\
& 4 \pi \sum_{n^{\prime}=0}^{\infty} \sum_{m^{\prime}=-n^{\prime}}^{n^{\prime}}(-1)^{n^{\prime}} i_{n^{\prime}}\left(\kappa\left|\overrightarrow{r_{0}}\right|\right) Y_{n^{\prime} m^{\prime}}^{*}\left(\widehat{r_{0}}\right) \times \\
& \times \sum_{N=\left|n-n^{\prime}\right|}^{n+n^{\prime}} \sum_{M=-N}^{N}(-1)^{N+M}\left[\frac{(2 n+1)\left(2 n^{\prime}+1\right)(2 N+1)}{4 \pi}\right]^{\frac{1}{2}} \times \\
& \times\left(\begin{array}{ccc}
n & n^{\prime} & N \\
0 & 0 & 0
\end{array}\right)\left(\begin{array}{ccc}
n & n^{\prime} & N \\
m & m^{\prime} & M
\end{array}\right) Y_{N,-M}(\widehat{d}) k_{N}(\kappa|\vec{d}|)
\end{aligned}
$$

sendo $\left(\begin{array}{lll}a & b & c \\ \alpha & \beta & \gamma\end{array}\right)=(-1)^{a-b-\gamma} \sqrt{\Delta(a b c)} \sqrt{(a+\alpha) !(a-\alpha) !(b+\beta) !(b-\beta) !(c+\gamma) !(c-\gamma) !}$

$$
\begin{aligned}
& \times \sum_{t}(-1)^{t}[t !(c-b+t+\alpha) !(c-a+t-\beta) !(a+b-c-t) !(a-t-\alpha) t(b-t+\beta) !]^{-1} e \\
& \Delta(a b c)=\frac{(a+b-c) !(b+c-a) !(c+a-b) !}{(a+b+c+1) !} .
\end{aligned}
$$

Substituindo a Equação (27) na Equação (26), obtém-se:

$$
\begin{aligned}
& U=\frac{32 k \pi}{\epsilon} \sum_{n=0}^{\infty} \sum_{m=-n}^{n} \sum_{n^{\prime}=0}^{\infty} \sum_{m^{\prime}=-n^{\prime}}^{n^{\prime}}(-1)^{n^{\prime}} i_{n^{\prime}}\left(k\left|\overrightarrow{r_{0}}\right|\right) \oint_{S_{0}} Y_{n^{\prime} m^{\prime}}^{*}\left(\widehat{r_{0}}\right) \sigma\left(\widehat{r_{0}}\right) d S_{0} \times \\
& \times \sum_{N=\left|n-n^{\prime}\right|}^{n+n^{\prime}} \sum_{M=-N}^{N}(-1)^{N+M}\left[\frac{(2 n+1)\left(2 n^{\prime}+1\right)(2 N+1)}{4 \pi}\right]^{\frac{1}{2}}\left(\begin{array}{ccc}
n & n^{\prime} & N \\
0 & 0 & 0
\end{array}\right) \times \\
& \times\left(\begin{array}{ccc}
n & n^{\prime} & N \\
m & m^{\prime} & M
\end{array}\right) Y_{N,-M}(\widehat{d}) k_{N}(k|\vec{d}|) i_{n}\left(k\left|\overrightarrow{r_{0}^{\prime}}\right|\right) \oint_{S_{0}^{\prime}} Y_{n m}^{*}\left(\widehat{r_{0}^{\prime}}\right) \sigma\left(\widehat{r_{0}^{\prime}}\right) d S_{0}^{\prime}
\end{aligned}
$$


Expandindo $\sigma\left(\widehat{r_{0}}\right)$ e $\sigma\left(\widehat{r_{0}^{\prime}}\right)$ em função dos Harmônicos Esféricos, tem-se que

$$
\begin{gathered}
\oint_{S_{0}} Y_{n^{\prime} m^{\prime}}^{*}\left(\widehat{r_{0}}\right) \sigma\left(\widehat{r_{0}}\right) d S_{0}=\oint_{S_{0}} Y_{n^{\prime} m^{\prime}}^{*}\left(\widehat{r_{0}}\right) \sum_{l=0}^{\infty} \sum_{p=-l}^{l} \sigma_{l p} Y_{l p}\left(\widehat{r_{0}}\right) d S_{0}= \\
\sum_{l=0}^{\infty} \sum_{p=-l}^{l} \sigma_{l p} \oint_{S_{0}} Y_{n^{\prime} m^{\prime}}^{*}\left(\widehat{r_{0}}\right) Y_{l p}\left(\widehat{r_{0}}\right) d S_{0}=\sum_{l=0}^{\infty} \sum_{p=-l}^{l} \sigma_{l p} \delta_{n^{\prime} l^{\prime}} \delta_{m^{\prime} p}=\sigma_{n^{\prime} m^{\prime}}
\end{gathered}
$$

e de forma análoga

$$
\begin{gathered}
\oint_{S_{0}^{\prime}} Y_{n m}^{*}\left(\widehat{r_{0}^{\prime}}\right) \sigma\left(\widehat{r_{0}^{\prime}}\right) d S_{0}^{\prime}=\oint_{S_{0}^{\prime}} Y_{n m}^{*}\left(\widehat{r_{0}^{\prime}}\right) \sum_{l=0}^{\infty} \sum_{p=-l}^{l} \sigma_{l p} Y_{l p}\left(\widehat{r_{0}^{\prime}}\right) d S_{0}^{\prime}= \\
=\sum_{l=0}^{\infty} \sum_{p=-l}^{l} \sigma_{l p} \oint_{S_{0}^{\prime}} Y_{n m}^{*}\left(\widehat{r_{0}^{\prime}}\right) Y_{l p}\left(\widehat{r_{0}^{\prime}}\right) d S_{0}^{\prime}=\sum_{l=0}^{\infty} \sum_{p=-l}^{l} \sigma_{l p} \delta_{n l} \delta_{m p}=\sigma_{n m}
\end{gathered}
$$

onde $\delta_{a b}$ é uma função delta de Kronecker, dada por $\delta_{a b}=0$ quando $a \neq b$ e $\delta_{a b}=1$ quando $a=b$.

Substituindo as Equações (29) e (30) na Equação (28), tem-se a expressão geral para a energia potencial eletrostática do problema considerado:

$$
\begin{gathered}
U=\frac{32 \kappa \pi}{\epsilon} \sum_{n=0}^{\infty} \sum_{m=-n}^{n} \sum_{n^{\prime}=0}^{\infty} \sum_{m^{\prime}=-n^{\prime}}^{n^{\prime}}(-1)^{n^{\prime}} i_{n^{\prime}}\left(\kappa\left|\overrightarrow{r_{0}}\right|\right) i_{n}\left(\kappa\left|\overrightarrow{r_{0}^{\prime}}\right|\right) \sigma_{n^{\prime} m^{\prime}} \sigma_{n m} \times \\
\times \sum_{N=\left|n-n^{\prime}\right|}^{n+n^{\prime}} \sum_{M=-N}^{N}(-1)^{N+M}\left[\frac{(2 n+1)\left(2 n^{\prime}+1\right)(2 N+1)}{4 \pi}\right]^{\frac{1}{2}}\left(\begin{array}{ccc}
n & n^{\prime} & N \\
0 & 0 & 0
\end{array}\right) \times \\
\times\left(\begin{array}{rrr}
n & n^{\prime} & N \\
0 & 0 & 0
\end{array}\right) Y_{N,-M}(\widehat{d}) k_{N}(k|\vec{d}|)
\end{gathered}
$$

\section{Considerações finais}

A escolha da equação diferencial parcial linearizada para descrição do sistema tratado nesse estudo foi motivada, antes de tudo, por apresentar grande simplicidade, uma vez que em tal descrição as hipóteses abrangem sistemas interessantes e o método da função de Green sempre pode ser utilizado.

A complexidade dos sistemas biológicos impede uma comparação direta dos resultados obtidos usando o modelo com o que ocorre no âmago celular, mas o modelo se mostrou factível, visto mostra que a interação entre uma superfície condutora e um aglomerado carregado é sempre atrativo, ao passo que glomerados com cargas de mesmo sinal é repulsivo. 
Dessa forma, o modelo de interação de um vírus com a membrana também está em acordo com os fenômenos da fagocitose e do brotamento (budding). O brotamento envolve uma organela recoberta por membrana com composição igual à da membrana plasmática e, portanto, com uma carga efetiva de mesmo sinal que resulta em uma interação eletrostática repulsiva.

Pode-se especular, ancorado nos resultados apresentados pelo modelo, que uma proteína com propriedades citolíticas, por exemplo fosfolipases de venenos, tem que apresentar uma carga efetiva resultante em $\mathrm{pH}$ da ordem de 7 para se aproximar da membrana celular, enquanto a fosfolipase eletricamente neutra não deve apresentar a capacidade de destruir membranas. Espera-se que o modelo possa elucidar tal questão em trabalhos futuros.

Os valores para a energia de interação e para a força entre a membrana e o vírus podem ser obtidos pelo modelo, que deverá oferecer a ordem de grandeza da energia e as distâncias em que tal interação é efetiva.

\section{Referências}

ALBERTS, Bruce; JOHNSON, Alexander; LEWIS, Julian; MORGAN, David; RAFF, Martin; ROBERTS, Keith; WALTER, Peter. Molecular biology of the cell. 6. ed. New York: Garland Science, 2015.

ATKINS, Peter; PAULA, Julia de; KEELER, James. Atkins Physical Chemistry. Oxford: OUP, 2018.

FESHBACH, Herman; MORSE, Philip M.; MICHIO, Masujima. Methods of Theoretical Physics. Mineola, NY: Dover Publications, 2019.

HILL, Terrell L. An Introduction to Statistical Thermodynamics. New York: Dover Publications, 1987.

JACKSON, John David. Classical Eletrodynamics. New York: John Wiley \& Sons, 1998.

MESSIAH, Albert. Mécanique Quantique. v. 2. Paris: Dunod, 1995.

MORAIS, D. M. G. Interações Eletrostáticas de Aglomerados Moleculares em Eletrólitos. 2005. 64 f. Dissertação (Mestrado em Biofísica Molecular) - Universidade Estadual Paulista, São Paulo, 2005.

TESO, Alexandre; DRIGO FILHO, Elso. AGOSTINHO NETO, Augusto. Solution of the PoissonBoltzmann equation for a system with four ionic species. Journal of Mathematcal Biology, v. 35, n. 7, p. 814-824, ago. 1997. DOI: https://doi.org/10.1007/s002850050078.

VERWEY, E. J. W.; OVERBEEK, J. T. G. Theory of the Stability of Lyophobic Colloids. New York: Elsevier Publishing Company, Inc., 1948. 